\title{
Magnetic field observations of low-mass stars
}

\author{
Ansgar Reiners \\ Universität Göttingen, Institut für Astrophysik, Friedrich-Hund-Platz 1, \\ D-37077 Göttingen, Germany \\ email: Ansgar.Reiners@phys.uni-goettingen.de
}

\begin{abstract}
Direct measurements of magnetic fields in low-mass stars of spectral class $M$ have become available during the last years. This contribution summarizes the data available on direct magnetic measurements in $\mathrm{M}$ dwarfs from Zeeman analysis in integrated and polarized light. Strong magnetic fields at kilo-Gauss strength are found throughout the whole M spectral range, and so far all field M dwarfs of spectral type M6 and later show strong magnetic fields. Zeeman Doppler images from polarized light find weaker fields, which may carry important information on magnetic field generation in partially and fully convective stars.
\end{abstract}

Keywords. Stars: activity - stars: late-type - stars: low-mass - brown dwarfs - stars: magnetic fields

\section{Introduction}

The existence and the topology of magnetic fields on low-mass stars is a topic of great interest. On the Sun, we know that magnetic fields are generated but strong fields are concentrated in small regions. Many mechanisms that could generate these fields were suggested, but their real physical nature still is a question of lively research (see other contributions in this volume). Rotation is probably one of the fundamental parameters that plays a key role in the generation of magnetic fields (e.g., Pizzolato et al. (2003); Reiners et al. (2009)). Other paramaters that could matter are the luminosity of the stars (Christensen et al. (2009)), the convective velocities, the size of the convection zone, and the existence of a region of great sheer between a radiative (probably rigidly rotating) core and an outer convective envelope, the so-called tachocline (Ossendrijver (2003)).

The general paradigm of the solar dynamo assumes that at least the cyclic part of the solar magnetic field is generated close to the tachocline through a so-called $\alpha \Omega$-type dynamo (but see also Brandenburg, this volume). Any dynamo mechanism that requires a tachocline must vanish in low-mass stars around spectral type M3 because this is the boundary beyond which stars no longer maintain a radiative core but remain fully convective during their entire lifetime (very young age, more massive stars are also fully convective). It is therefore particularly interesting to investigate magnetic fields around the mass boundary between partially and fully convective stars.

\section{Methods to measure magnetic fields}

Magnetic fields are measured with different techniques. Here, we consider only "direct" measurements of magnetic fields, i.e., we are not considering magnetic fields that are inferred through the observation of secondary indicators. Such indicators are very useful because they trace non-thermal radiation processes, which are probably connected to the existence of magnetic fields (most prominent tracers are chromospheric Ca H\&K and $\mathrm{H} \alpha$ emission, e.g., Hartmann \& Noyes (1987), Mohanty \& Basri (2003; coronal 
X-ray emission, e.g., Pizzolato et al. (2003); or radio emission from high-energy electrons, Berger (2006), Hallinan et al. (2008)).

Direct observations of magnetic fields can be accomplished through observation of the Zeeman effect, which shows direct consequences of magnetic fields on the appearance of spectral features. Principally, two different ways must be distinguished. The first method is the measurement of the Zeeman effect in polarized light, usually detecting circular polarization in Stokes V. This method can detect relatively weak fields because it is a differential method that can be calibrated rather accurately. One caveat is that only a net polarization can be detected. Polarization signals even of strong magnetic fields can cancel out each other if they are distributed in small entities. Another problem of measurements in Stokes $\mathrm{V}$ is that only a fraction of the light can be used so that the usually a number of simplifications have to be applied. The second method is to determine the magnetic fields strength from the appearance of spectral lines in Stokes I, i.e., from integrated light without any polarization analysis. Here, the main problem is that the effect of Zeeman broadening is relatively small in comparison to other broadening effects in spectral lines so that calibration is very difficult and leads to large uncertainties. On the other hand, this method can detect the total mean unsigned magnetic flux including components that are distributed in small cells of opposite polarity.

\section{A compilation of results}

This article gives an overview of recent (direct) magnetic field measurements in lowmass stars of spectral class M. Today, the number of measurements is constantly growing and we can start to compare results from different techniques in the same stars. This opens another channel of investigation because the differences between the results from different techniques carries information about the magnetic fields and their distribution.

\subsection{Stokes $I$}

Measurements of stellar magnetic fields in integrated light, Stokes I, can be carried out by calculating the polarized radiative transfer in magnetically sensitive spectral lines and comparing the models to observations. Robinson (1980), has introduced the method to measure stellar magnetic fields in spectral absorption lines. This method was used by several groups, mostly in atomic spectral lines in the optical wavelength range. A summary of this effort can be found in Saar (1996), and Saar (2001). Johns-Krull \& Valenti (2000), summarize results on magnetic field measurements including spectral lines in the infrared wavelength range. These authors center on developments for fully convective $\mathrm{M}$ dwarfs and pre-main sequence stars. In very cool atmospheres, e.g., in mid-M dwarfs at the boundary to full convection, the usefulness of atomic lines becomes limited because atomic lines are being buried under the haze of molecular absorption bands.

Valenti \& Johns-Krull (2001), discuss molecular absorption bands of FeH as a Zeeman diagnostic for very cool dwarfs. FeH shows a series of narrow, strong absorption lines for example around $1 \mu \mathrm{m}$, a spectral region where the spectral energy distribution of $\mathrm{M}$ dwarfs is close to its maximum. A problem for magnetic field measurements with $\mathrm{FeH}$ is that magnetic splitting is difficult to calculate. Reiners \& Basri (2006), introduced a semiempirical approach in which the magnetic field of an M-star is measured by comparison of its $\mathrm{FeH}$ lines to $\mathrm{FeH}$ lines observed in stars of known magnetic field. The magnetic fields of these template stars have to be measured in atomic lines, hence only early-M stars can be used for this method. Reiners \& Basri (2006), show that FeH absorption in $\mathrm{M}$ dwarfs follows an optical-depth scaling that allows to use early-M template spectra to 
Table 1. Stokes I measurements of magnetic flux among M dwarfs.

\begin{tabular}{|c|c|c|c|c|}
\hline Name & Spectral Type & $\begin{array}{r}v \sin i \\
{\left[\mathrm{~km} \mathrm{~s}^{-1}\right]}\end{array}$ & $\begin{array}{r}B f \\
{[\mathrm{kG}]}\end{array}$ & Ref \\
\hline Gl 182 & M0.5 & 9 & 2.5 & $e$ \\
\hline Gl $494 \mathrm{~A}$ & M0.5 & 11 & 3.0 & $e, f$ \\
\hline Gl 70 & M2.0 & $\leqslant 3$ & 0.0 & $a$ \\
\hline Gl 569A & M2.5 & $\leqslant 3$ & 1.8 & $e$ \\
\hline Gl 729 & M3.5 & 4 & 2.2 & $a, b$ \\
\hline Gl 873 & M3.5 & $\leqslant 3$ & 3.9 & $a, b$ \\
\hline Gl 388 & M3.5 & $\approx 3$ & 2.9 & $a, b$ \\
\hline GJ 3379 & M3.5 & $\leqslant 3$ & 2.3 & $c$ \\
\hline Gl 876 & M4.0 & $\leqslant 3$ & 0.0 & $a$ \\
\hline GJ $1005 \mathrm{~A}$ & M4.0 & $\leqslant 3$ & 0.0 & $a$ \\
\hline GJ 2069B & M4.0 & 6 & 2.7 & $c$ \\
\hline GJ 299 & $\mathrm{M} 4.5$ & $\leqslant 3$ & 0.5 & $a$ \\
\hline GJ 1227 & M4.5 & $\leqslant 3$ & 0.0 & $a$ \\
\hline GJ 1224 & M4.5 & $\leqslant 3$ & 2.7 & $a$ \\
\hline Gl 285 & M4.5 & 5 & $>3.9$ & $a, b$ \\
\hline Gl 493.1 & M4.5 & 18 & 2.1 & $c$ \\
\hline LHS 3376 & M4.5 & 19 & 2.0 & $c$ \\
\hline Gl 905 & M5.0 & $\leqslant 3$ & 0.0 & $a$ \\
\hline GJ 1057 & M5.0 & $\leqslant 3$ & 0.0 & $a$ \\
\hline GJ $1154 \mathrm{~A}$ & M5.0 & 6 & 2.1 & $c$ \\
\hline GJ 1156B & M5.0 & 17 & 2.1 & $c$ \\
\hline LHS $1070 \mathrm{~A}$ & M5.5 & 8 & 2.0 & ${ }^{c}$ \\
\hline GJ 1245B & M5.5 & 7 & 1.7 & $a$ \\
\hline GJ 1286 & M5.5 & $\leqslant 3$ & 0.4 & $a$ \\
\hline GJ 1002 & M5.5 & $\leqslant 3$ & 0.0 & $a$ \\
\hline Gl 406 & M5.5 & 3 & $2.1-2.4$ & $a, d$ \\
\hline GJ 1111 & M6.0 & 13 & 1.7 & $a$ \\
\hline GJ $412 B$ & M6.0 & 5 & $>3.9$ & $c$ \\
\hline VB 8 & M7.0 & 5 & 2.3 & $a$ \\
\hline LHS 3003 & M7.0 & 6 & 1.5 & $a$ \\
\hline LHS 2645 & M7.5 & 8 & 2.1 & $a$ \\
\hline LP 412-31 & M8.0 & 9 & $>3.9$ & $a$ \\
\hline VB 10 & M8.0 & 6 & 1.3 & $a$ \\
\hline LHS $1070 \mathrm{~B}$ & M8.5 & 16 & 4.0 & $c$ \\
\hline LHS $1070 \mathrm{C}$ & M9.0 & 16 & 2.0 & $c$ \\
\hline LHS 2924 & M9.0 & 10 & 1.6 & $a$ \\
\hline LHS 2065 & M9.0 & 12 & $>3.9$ & $a$ \\
\hline
\end{tabular}

${ }^{a}$ Reiners \& Basri (2007), ${ }^{b}$ Johns-Krull \& Valenti (2000), ${ }^{c}$ Reiners et al. (2009), ${ }^{d}$ Reiners et al. (2007), ${ }^{e}$ Reiners \& Basri (2009), ${ }^{f}$ Saar (1996)

measure magnetic fields even in very-late type $\mathrm{M}$ dwarfs. Using this method, Reiners \& Basri (2007), carried out the first survey of magnetic field measurements in M dwarfs, and Reiners et al. (2007), and Reiners \& Basri (2009), applied the same method to another set of stars. The uncertainty of their magnetic field measurements is on the order of a kilo-Gauss so that small fields of a few hundred Gauss are difficult to detect. The method was successfull in detecting strong (several kilo-Gauss) fields in many M dwarfs, in particular all M dwarfs with spectral type M6 and later show strong magnetic fields.

The current sample of $\mathrm{M}$ dwarfs with measured magnetic fields is collected in Table 1. Strong magnetic fields are found throughout the whole range of spectral subclasses M0M9. More specific, among the early-M stars with spectral type M0-M5.5, magnetic flux 

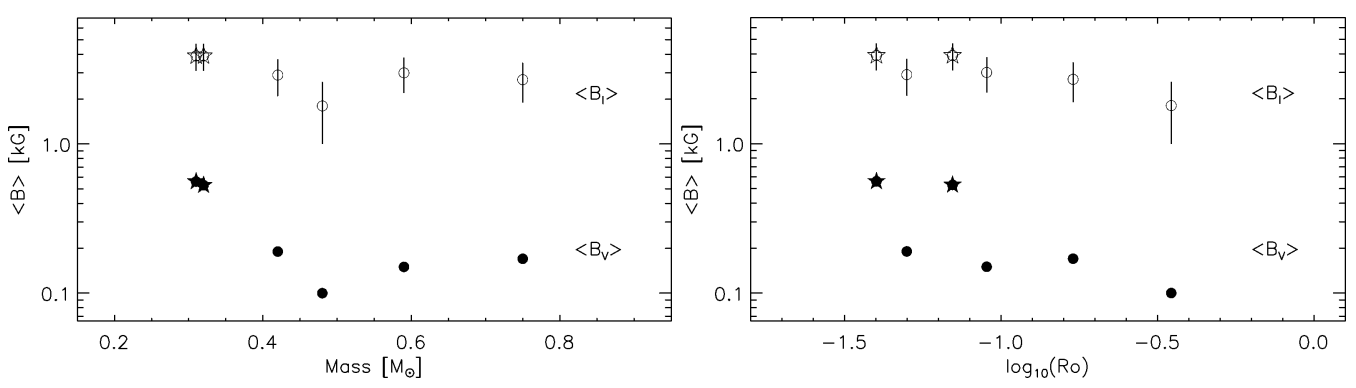

Figure 1. Mean magnetic field measurements from Stokes I (open symbols) and Stokes V (filled symbols) as a function of Mass (left panel) and Rossby number (right panel).

is found between 0 and $4 \mathrm{kG}$. Magnetic flux scales with rotation (and activity in $\mathrm{H} \alpha$ scales with magnetic flux), which is expected because of the well-known rotation-activity connection among warmer stars (e.g., Pizzolato et al. (2003)). The meaning of this is threefold: 1) M dwarfs (partially as well as fully convective) can generate strong magnetic fields; 2) Stellar activity is of magnetic origin; 3) The connection between rotation and magnetic field generation / magnetic activity also holds in early- to mid-M dwarfs.

Magnetic field measurements have also been carried out in young low-mass stars (see for example Johns-Krull (2007)). Measurements of magnetic fields in young objects are the subject of Johns-Krull (2009), in this volume.

\subsection{Stokes $V$}

The method of Zeeman Doppler Imaging in Stokes V has been very successfull during the last years providing the first information on stellar magnetic structure (e.g., Donati et al. (1997)). The somewhat surprising result of axisymmetric magnetic topology in a fully convective star was reported by Donati et al. (2006) using this technique. Recently, Donati et al. (2008), and Morin et al. (2008), measured magnetic fields in Stokes V in a sample of early- and mid-M dwarfs on both sides of the boundary to full convection. They find magnetic flux on the level of a few hundred Gauss in their sample stars. An important result of this work is that the strength of magnetic flux and the topology of magnetic fields show a marked change at spectral class M3 where stars are believed to become fully convective. This is very interesting because it was expected that magnetic fields in fully convective stars (Durney et al. (1993)) are less organized than in stars with a radiative core that might generate large-scale dipolar fields through an interface dynamo located at the tachocline (Charbonneau (2005); Ossendrijver (2003)).

\section{A comparison of results}

With the growing number of magnetic field measurements in both, integrated light and Zeeman Doppler Imaging from Stokes V, it becomes possible to compare results from both techniques. This may shed more light on the topology of M star magnetic fields because both techniques are sensitive to different aspects of the flux: While in Stokes I the mean total unsigned flux is observed, Stokes V is sensitive to the net polarized flux, i.e., to magnetic field distributions that lead to a net polarization signal in time series taken for the Doppler Images.

Currently, six M dwarfs have magnetic flux measurements from both Stokes V and Stokes I. These results are shown in Fig. 1 as a function of mass and Rossby number. In all cases, the magnetic flux measured from Stokes I is much stronger than the value from Zeeman Doppler Imaging. This is not surprising, as mentioned above, because the former 
is sensitive to the total flux while the latter normally is not. The ratio between magnetic flux in Stokes $\mathrm{V}$ and $\mathrm{I}$ is between 1:7 and 1:17, this means the fraction of magnetic flux seen in Stokes $\mathrm{V}$ is between $6 \%$ and $15 \%$.

\section{Summary}

Magnetic field measurements are becoming available for a growing number of low-mass stars through analysis of integrated and polarized light. A compilation of results from Stokes I measurements in the FeH molecule used among M dwarfs is given in this article. Other techniques are available probing different aspecs of the magnetic field distribution and making different assumptions.

Generally, fields of kilo-Gauss strength are ubiquitously found in late-M dwars, which is consistent with their activity. Magnetic field topology is important to answer the question about the underlying dynamo mechanism, and first results on topologies are available from Zeeman Doppler imaging finding predominantly axisymmetric geometries in fully convective stars. However, the applied techniques are probably missing a substantial fraction of the total magnetic flux so that the full field distribution may not be visible.

Brown dwarfs are generally rapidly rotating and so far no useful tracer for Zeeman analysis was found (at least in old brown dwarfs), which poses a serious problems to the detection of magnetism. Whether brown dwarfs can maintain strong magnetic fields is an open question, but so far there seems to be no reason to believe they wouldn't.

I acknowledge funding through a DFG Emmy Noether Fellowship (RE1664/4-1).

\section{References}

Berger, E., 2006, ApJ 648, 629

Charbonneau, P., 2005, Living Reviews in Solar Physics 2, 2

Christensen, U., Holzwart, V., \& Reiners, A., 2009, Nature, in press

Donati, J.-F., Semel, M., Carter, B. D., Rees, C. E., \& Cameron, A. C., 1997, MNRAS 291, 658

Donati, J.-F., Forveille, T., Cameron, A. C., et al., 2006, Science 311, 633

Donati, J.-F., Morin, J., Petit, P., et al., 2008, MNRAS 390, 545

Durney, B. R., de Young, D.S., \& Roxburgh, I. W., 1993, SP 145, 207

Hallinan, G., Antonova, A., Doyle, J. G., et al., 2008, ApJ 684, 644

Hartmann, L. W. \& Noyes, R. W., 1987, ARAA 25, 271

Johns-Krull, C. \& Valenti, J. A., 2000, ASP Conf. Ser., 198, 371

Johns-Krull, C. M., 2007, ApJ 664, 975

Johns-Krull, C. M., 2009, this volume

Mohanty, S. \& Basri, G., 2003, ApJ 583, 451

Morin, J., Donati, J.-F., Petit, P., et al., 2008, MNRAS 390, 567

Ossendrijver, M., 2003, A\&AR 11, 287

Pizzolato, N., Maggio, A., Micela, G., Sciortino, S., \& Ventura, P., 2003, A $\& A$ 397, 147

Reiners, A. \& Basri, G., 2006, ApJ 644, 497

Reiners, A. \& Basri, G., 2007, ApJ 656, 1121

Reiners, A., Schmitt, J. H. M. M., \& Liefke, C. 2007, A\&SA 466, L13

Reiners, A., Basri, G., \& Browning, M., 2009, ApJ, in press, arXiv.org:0810.5139

Reiners, A. \& Basri, G., 2009, submitted

Robinson, R. D., 1980, ApJ 239, 961

Saar, S. H., 1996, in IAU Symp. 176, Stellar Surface Structure, Strassmeier, K. G., Linsky, J. L. (eds.), Kluwer, p.237

Saar, S. H., 2001, ASP Conf. Ser. 223, 292

Valenti, J. A., \& Johns-Krull, C., 2001, ASP Conf. Ser. 248, 179 


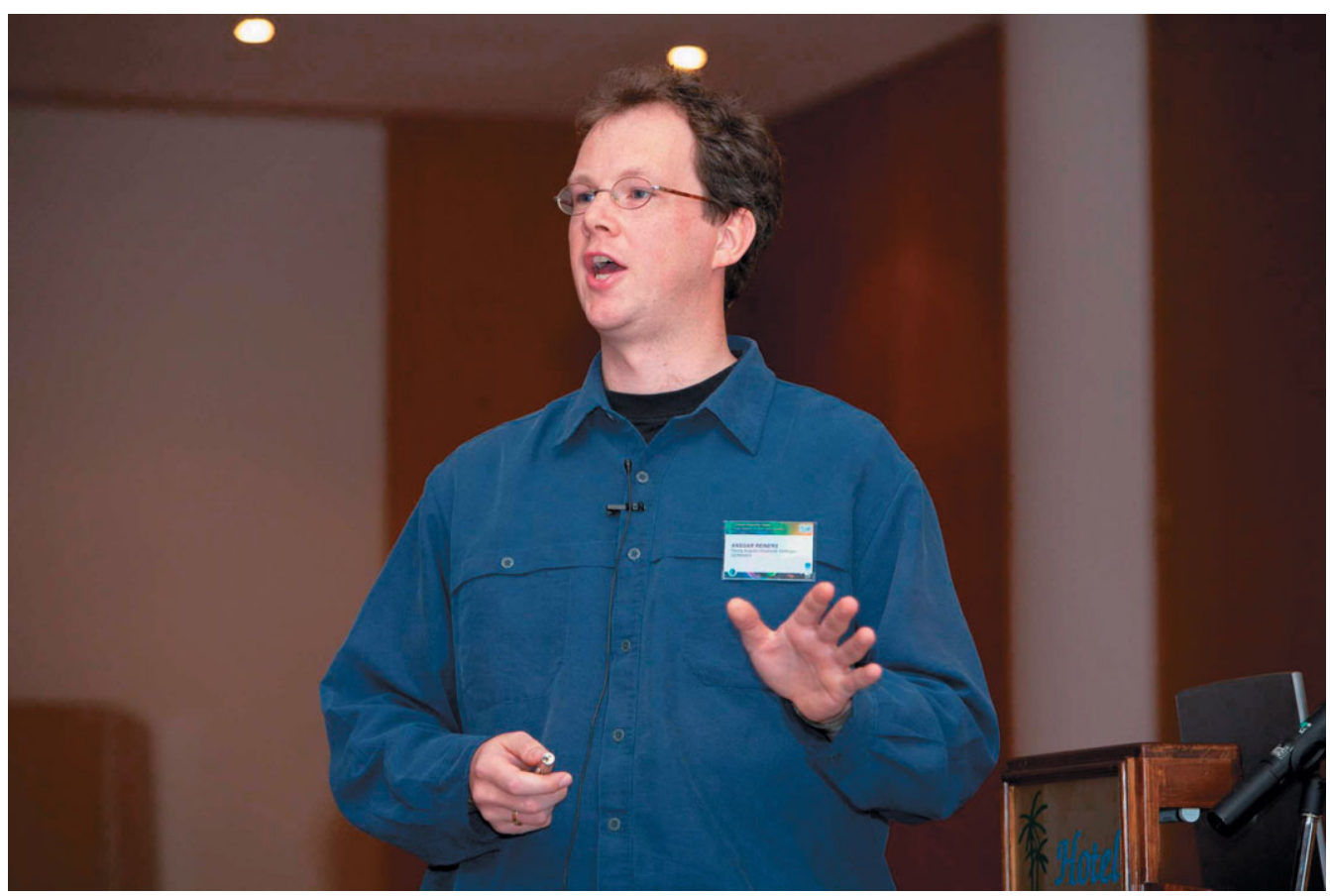

Ansgar Reiners

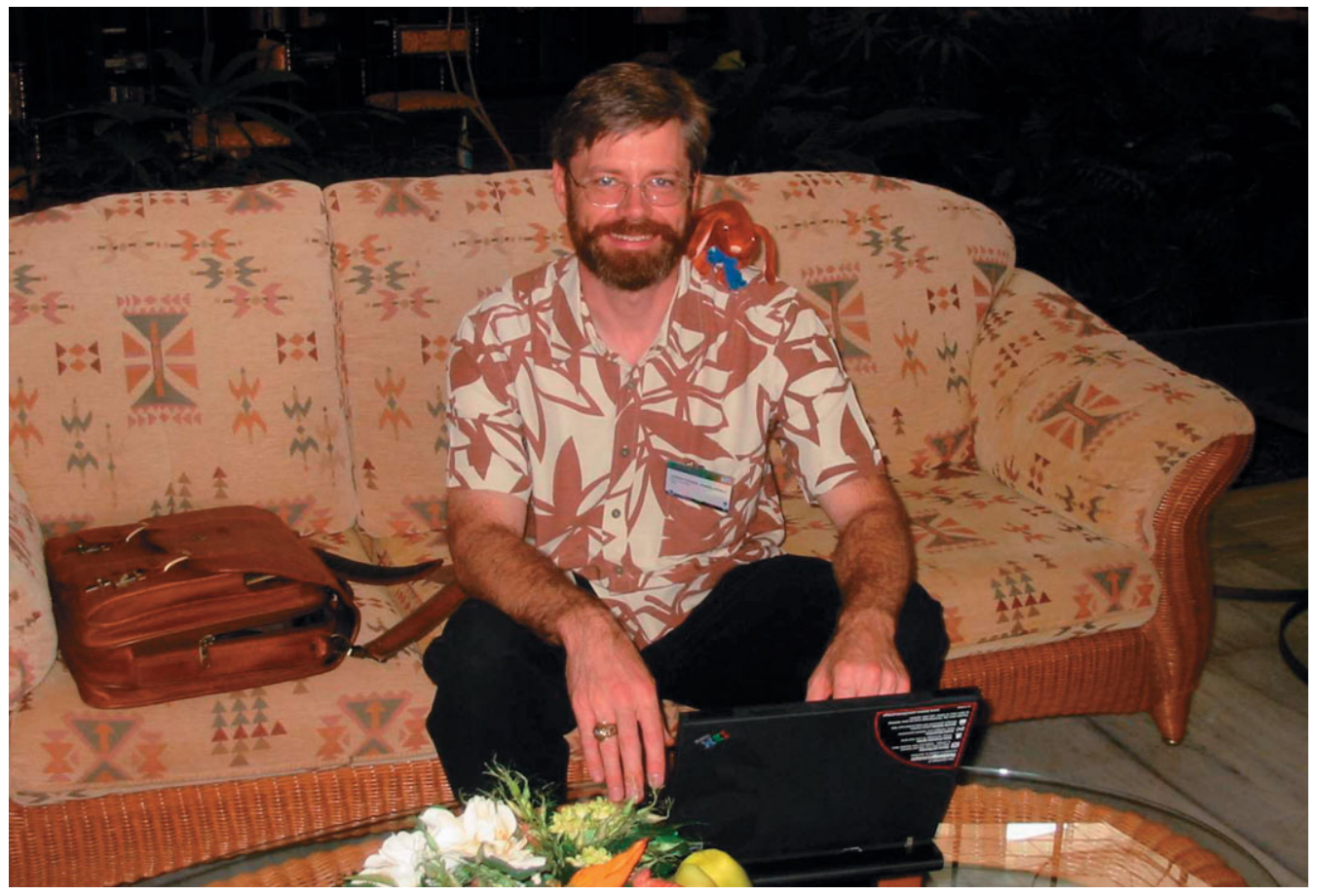

Christopher Johns-Krull 